\title{
Incidencia de las rutas del transporte público en la contaminación ambiental microbiana de buses urbanos.
}

Incidence of public transport routes in the environmental pollution, microbial of urban buses.

Ing. Raúl Fernando Galarza Chacón $\mathrm{Mg}^{1}$, Ing. Segundo Manuel Espín-Lagos $\mathrm{Mg}^{2}$, Ing. Francisco Zaldumbide-Ortiz $\mathrm{MSc}^{3}$, Ing. Oscar Fabián Tene Salazar ${ }^{4}$, BQF. Isabel Cristina López-Villacís Mg. ${ }^{5}$.

Recibido: 07-12-2017 / Revisado: 04-02-2018 Aceptado: 21-03-2018/ Publicado: 01-04-2018

\begin{abstract}
.
DOI: $\underline{\text { https://doi.org/10.33262/cienciadigital.v2i2.76 }}$

Urban transport units in suitable environmental conditions are an ideal site for the growth of bacteria. The research was based on the exploratory study with the validation of the problem of microbiological environmental contamination by qualitative and quantitative analysis of the inert surfaces of the buses that are in direct contact with the hands of the people. The methodology included the use of validated methods for the collection and analysis of samples, such as, swabbing of surfaces, culture of microorganisms in general and specific media, Microgen ID A $+\mathrm{B}$ and petrifilm plates for confirmation and quantification of a specific microorganism. The results showed that 9 of 10 units have some type of bacterium. Also, there is a prevalence of Staphylococcus aureus with $57.69 \%$, then the quantitative analysis was performed, which showed that the most contaminated place of the bus is the vertical handle of the stop button, followed by the handle and the seat base (391.76, 333.46, 228.18 colony forming units per square centimeter ), respectively. The hypothesis showed that buses that circulate in hospital areas have higher levels of microbial environmental contamination.

${ }^{1}$ H.G.P. de Tungurahua, Ecuador, raul.galarza@tungurahua.gob.ec

${ }^{2}$ Universidad Técnica de Ambato, Ecuador, sespin@uta.edu.ec

${ }^{3}$ Consultoría y Servicios Ambientales. CONSECAM, Ecuador, fxzo@yahoo.es

${ }^{4}$ Universidad Técnica Ambato, Centro de Apoyo de Desarrollo Metalmecánico. Ecuador, of.tene@uta.edu.ec ${ }^{5}$ Universidad Técnica de Ambato, Ecuador, ic.lopez@uta.edu.ec
\end{abstract}


Keywords: Urban Transport, Microbiological Contamination, Buses, Staphylococcus Aureus.

\section{Resumen.}

Las unidades de transporte urbano en condiciones ambientales adecuadas son consideradas un sitio ideal para el crecimiento de bacterias. La investigación parte del estudio exploratorio con la validación del problema de la contaminación ambiental microbiológica mediante análisis cualitativo y cuantitativo de las superficies inertes de los buses que están en contacto directo con las manos de los usuarios. La metodología contempló la utilización de métodos validados para la toma y análisis de las muestras, tales como: hisopado de superficies, cultivo de microorganismos en medios generales y específicos, Microgen y placas Petri film para confirmación y cuantificación de un microorganismo específico. Los resultados arrojaron que 9 de cada 10 unidades poseen algún tipo de bacteria, existiendo prevalencia del Staphylococcus aureus con el 57,69\%; el lugar más contaminado del bus es el asidero vertical de botón de parada, seguido del asidero y base del asiento $(391,76$; 333,46; 228,18 unidades formadoras de colonia por centímetro cuadrado) respectivamente. La hipótesis demostró que los buses que circulan por áreas de hospitales tienen niveles mayores de contaminación ambiental microbiana.

Palabras Claves: Transporte Urbano, Contaminación Microbiológica, Buses, Staphylococcus Aureus.

\section{Introducción.}

La investigación cobra importancia debido a que la tercera causa de muerte a nivel del Ecuador es ocasionada por la influenza y enfermedades respiratorias asociadas a diferentes patógenos, siendo la población más susceptible a contraer estas enfermedades los niños y adultos mayores. La motivación está alineada al Plan Nacional del Buen Vivir en su objetivo número 3 relacionado a temas de Salud Pública.

En el mundo se presenta una gran inclinación al transporte público urbano ya sea por economía, rapidez o también por preferencia al momento de agilizar su traslado de un punto a otro; en diversos países desarrollados este tipo de transporte es muy útil ya que este medio de transporte no descansa ni un solo instante y brinda un servicio excelente a la ciudadanía, pero la parte importante se da al trabajar en las noches como es el caso en España, Estados Unidos, Brasil, entre otros (Kassen, 2009). 
Investigaciones recientes confirman que el transporte urbano puede ser un reservorio para transmisiones de Staphylococcus, las transmisiones cruzadas por Staphylococcus pueden ser de varias fuentes, incluyendo hospitales, comunidades y ganado (Roberts, 2013).

Durante las horas pico en particular, los autobuses públicos pueden llegar a ser muy congestionados (Gaymard et al, 2016). La aglomeración puede proporcionar las condiciones de calor y humedad internas que las bacterias y los gérmenes necesitan para su desarrollo, que causa problemas de higiene para los pasajeros (Lutz et al, 2014). Casi la mitad (49,3\%) de los usuarios de autobuses están preocupados por la presencia de bacterias en los puntos de contacto dentro de un autobús, tales como barandillas, tejidos de los asientos y los botones" (Yang et al, 2015). Las superficies de contacto en entornos con un gran tránsito de masas suponen un reservorio óptimo para los microbios infecciosos. Las condiciones de aglomeración en lugares cerrados favorecen la contaminación (Conceicao et al, 2013) por bacterias entre miles de personas que se movilizan diariamente en la ciudad de Ambato. El Estaphylococos aureus puede sobrevivir por largos períodos en los objetos inanimados, lo que puede representar un reservorio importante para su difusión (French \& Otter, 2009). Las patologías respiratorias pueden atacar directamente a grupos vulnerables como niños, adultos mayores y mujeres embarazadas sobre todo en ambientes de alta densidad de usuarios como medios de transporte urbano, hospitales, mercados, etc.

\section{Materiales y métodos.}

Recolección de muestras ambientales: Debido a la inexistencia de una normativa nacional o internacional que explique la metodología que indique los lugares y número de tomas de muestras en el interior del bus, número, hora y día de toma de muestras, se planteó un protocolo que permite replicar investigaciones similares:

Número de muestras tomadas en el interior del bus: Se realizó un muestreo no aleatorias por duplicado en el asidero vertical de aviso de parada, asidero y base del asiento, se seleccionaron debido a que son los lugares con los más altos niveles de contacto manual con las superficies inanimadas en el interior del bus (Coia, 2006).

Delimitación temporal: La investigación se realizó entre los meses de octubre 2016 octubre 2017.

Horario de toma de muestras: El monitoreo se efectuó en la noche, siendo variante entre 6 a 10 de la noche dependiendo de la cooperativa y en función del último recorrido de cada una de las unidades de transporte antes de cualquier limpieza (acumulación de bacterias durante el día), la metodología que se utilizó contempló las condiciones más adversas del ambiente tanto para el día como para la hora (Lutz et al, 2014). 
Día de toma de muestras: El muestreo se realizó el día lunes, por ser el día de mayor afluencia de personas a la ciudad de Ambato, este criterio de muestreo se seleccionó tomando en cuenta condiciones extremas de contacto de usuarios con los lugares contaminados, además de la interacción entre personas de las zonas urbanas y rurales en el interior de los buses.

Registro de condiciones ambientales en el interior y exterior del bus: La temperatura y humedad relativa ambiental, y las condiciones ambientales en el interior del bus, la toma de muestra en las superficies se realizó con la utilización del medidor de condiciones ambientales Kestrel 3500.

Selección de rutas: Se eligieron las rutas de los autobuses que servían a los principales hospitales y las que no, se seleccionan en función de la mayor demanda en número de pasajeros. Formalmente, el cantón tiene un registro de dos hospitales generales: el Hospital General Ambato y el Hospital IESS, ubicados en la cabecera cantonal; mientras que se ha considerado como hospital básico al Municipal Nuestra Señora de la Merced.

Método para la toma de muestras: La toma de muestras se realizó mediante el método de hisopado de superficies por ser la metodología recomendada para lugares que tienen superficies que no son planas, posteriormente se siembra cada una de ellas en agar (French \& Otter, 2009).

Aislamiento e identificación: Se realizó en medios de Agar sangre y MacConkey, se utilizó pruebas bioquímicas, además el método de Microgen para identificar bacterias que no son comunes.

Utilización de métodos válidos para análisis de laboratorio: La realización de los análisis se efectuó con la aplicación de medidas de control ambiental determinados por los estándares de laboratorio de microbiología.

Fase cualitativa y cuantitativa: En la fase cualitativa se identificó y caracterizó las bacterias en el interior de los buses, luego se cuantificó los niveles de concentración de cada una en los sitios determinados del muestreo.

Población de estudio: La población de estudio considerada para la investigación está formada por las operadoras de transportes que permiten la movilidad de todas las personas, dentro de la ciudad de Ambato transitan 403 unidades diariamente, las operadoras que se encuentran autorizadas para circular son las siguientes: Jerpazsol, Unión Ambateña, Tungurahua, Libertadores y Vía Flores las cuales son consideradas como trasporte urbano cubren diferentes rutas para satisfacer las necesidades de los habitantes debido a la demanda que se genera día tras día, lo que se presenta en la Tabla 1. 
Tabla 1. Población de las Unidades de transporte urbano de Ambato

\begin{tabular}{lc}
\hline \multicolumn{1}{c}{ Población de estudio } & \# de Vehículos por operadora \\
\hline Operadora Tungurahua & 144 \\
Operadora Vía Flores & 45 \\
Operadora Unión Ambateña & 98 \\
Operadora Jerpazsol & 51 \\
Operadora Los Libertadores & 65 \\
TOTAL & $\mathbf{4 0 3}$ \\
\hline
\end{tabular}

Fuente: Dirección de Tránsito, Transporte y Movilidad.

Muestreo: Se determinó el tamaño de la muestra aplicando la fórmula para cálculo de la muestra para poblaciones finitas, se estableció un nivel de confianza del $95 \%$ y error del 5\%, el tamaño de la muestra fue de 78 buses urbanos. Para la determinación del número de vehículos que serán muestreados se utilizó un muestreo probabilístico estratificado proporcional indicado en la Tabla 2, el que permitió obtener el número exacto de unidades por operadora.

Tabla 2: Número de vehículos que se deben muestrear por operadora.

\begin{tabular}{lll}
\hline \multicolumn{1}{c}{ Cooperativa } & \multicolumn{1}{c}{ \% } & \multicolumn{1}{c}{$\#$} \\
\hline Tungurahua & 35,73 & 28 \\
Vía Flores & 11,17 & 9 \\
Unión Ambateña & 24,32 & 19 \\
Jerpazsol & 12,66 & 10 \\
Los Libertadores & 16,13 & 12 \\
& Total & 78 \\
\hline
\end{tabular}

Fuente: Grupo de investigación.

Se aplicó un muestreo probabilístico estratificado proporcional para la determinación del número de unidades por cooperativa. \%: Porcentaje que representa cada cooperativa del total; \#: Número de buses para el muestreo.

Zonas de toma de muestras: En la Figura1, se ilustran los lugares definidos para la toma de muestras en un bus. 
Figura 1: Selección de puntos para la toma de muestras en el interior del bus.

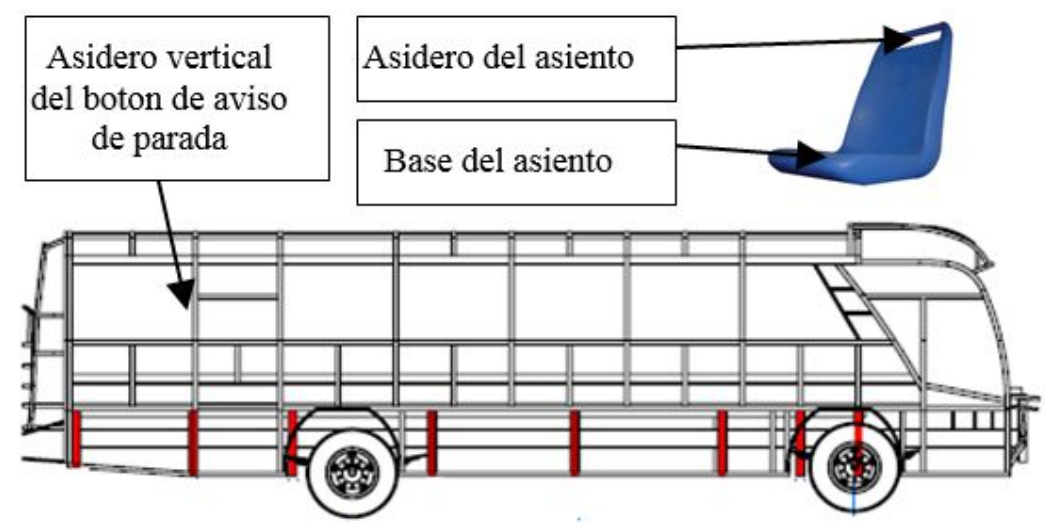

Fuente: Grupo de investigación.

Conservación y transporte de la muestra: Se colocaron en un contenedor isotérmico, de tal manera de asegurar que la temperatura del contenedor no sea mayor de $14^{\circ} \mathrm{C}$, a fin de asegurar la vida útil de la muestra hasta su llegada al laboratorio. El tiempo de transporte entre la toma de muestra y la recepción en el laboratorio estará en función estricta de dicha temperatura, no debiendo exceder las 24 horas y excepcionalmente las 36 horas.

Diseño experimental y estadístico de la investigación: se basó en el análisis estadístico para comparación de 2 muestras independientes, como se muestra en la Tabla 3, se estudió si existe relación entre el valor medio de una variable respuesta o característica (nivel de contaminación microbiana) y una variable cualitativa (Bus circula o no por un hospital).

Tabla 3: Diseño experimental para comparación de 2 muestras independiente.

\begin{tabular}{lll}
\hline & Variable dicotómica $^{\text {a }}$ \\
\hline Variable cuantitativa & Circula por un hospital & No circula por un hospital \\
M1 & - & - \\
M2 & - & - \\
Mn-1 & - & - \\
Mn & - & - \\
\hline
\end{tabular}

Fuente: Grupo de Investigación.

M1 hasta Mn son valores de las repeticiones de las mediciones determinadas de acuerdo al muestreo. a Se determina de acuerdo a las rutas que cada una de las cooperativas da el servicio. 
Valores máximos permisibles para bacterias patógenas: "Las superficies inertes que tengan calidad sanitaria aceptable deben tener valores menores a 400 unidades formadoras de colonia por centímetro cuadrado (NOM-093-SSA1, 1994).

Hipótesis general de la investigación: Se plantea el siguiente enunciado como hipótesis de la investigación: "En las rutas de transporte urbano que circulan por áreas de hospitales existe contaminación ambiental en los asideros de los buses de la ciudad de Ambato".

\section{Resultados y discusión.}

Debido a la complejidad de la investigación se trató de controlar y cuantificar todos los factores externos (variables exógenas) que influyen en la variable de respuesta (contaminación ambiental microbiológica en la superficie), tales como la ruta (lugares por donde circulan las unidades), hora y día de toma de muestras, lugar de la toma de muestras pueden tener incidencia en la incertidumbre de los resultados obtenidos.

Resultados investigación cualitativa: Para verificar y validar el problema de contaminación ambiental de las superficies de los transportes urbanos se realizó un screening inicial, para esto se planificó la toma de muestras de los asideros verticales del botón de aviso de parada, debido a que este lugar es el de mayor contacto de las personas con superficies inanimadas del bus, cuyos resultados se evidencian en la Tabla 4.

Tabla 4: Frecuencia de bacterias identificadas en el interior del bus.

\begin{tabular}{|c|c|c|}
\hline Bacteria & Frecuencia & Porcentaje \\
\hline Staphylococcus aureus & 45 & 57,69 \\
\hline Staphylococcus epidermidis & 15 & 19,23 \\
\hline No hay crecimiento & 8 & 10,25 \\
\hline Klebsiella pneumoniae & 5 & 6,41 \\
\hline Escherichia coli & 5 & 6,41 \\
\hline Total & 78 & 100,00 \\
\hline
\end{tabular}

Fuente: Grupo de investigación.

\section{Resultados investigación cuantitativa:}

En la Figura 3, se presenta la planificación para el monitoreo ambiental cuantitativa en el interior de los buses. 
Figura 3: Valores promedios por cada lugar de toma de muestra.

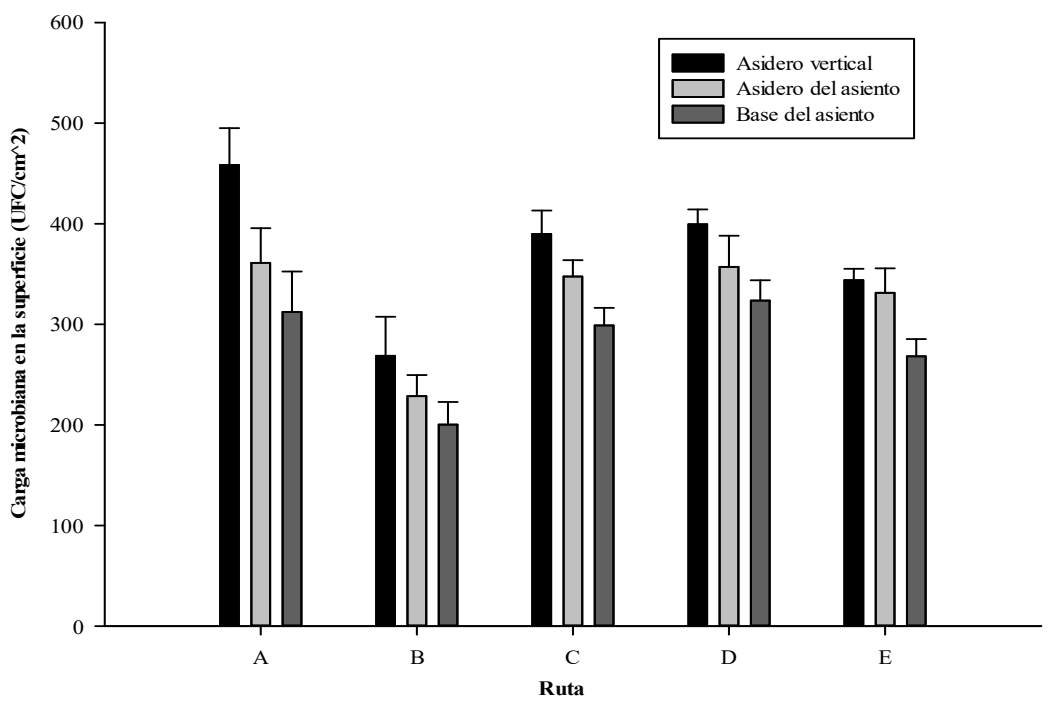

Fuente: Grupo de investigación.

Donde:

A: Ficoa - Terremoto - Totoras.

B: La Florida - 4 Esquinas - Cashapamba.

C: La Joya - Cdla Militar.

D: Los Ángeles.

E. Juan Benigno Vela - Redondel Izamba.

Análisis inferencial entre el lugar de la toma de muestra en el interior del bus y la carga microbiana en la superficie: Sirvió para comparar varios grupos en una variable cuantitativa. Se trata, por tanto, de una generalización de la Prueba de T para dos muestras independientes al caso de diseños con más de dos muestras, como se evidencia en la tabla 5.

Tabla 5: Prueba ANOVA de un factor lugar de toma de muestra vs. Carga microbiana.

\begin{tabular}{lll}
\hline F.V. & F & p valor \\
\hline Modelo & 91,06 & $<0,0001$ \\
Lugar de la toma de la muestra & 91,06 & $<0,0001$ \\
\hline
\end{tabular}

Fuente: Grupo de investigación.

Se aplicó un nivel de significancia $=0,05$. F.V.: Fuentes de variación, F: Estadístico F, p valor: Probabilidad de que Ho sea verdadera.

En el análisis de ANOVA de un factor el nivel de significancia de 0,0001es menor a 0.05 , esto quiere decir que se acepta la hipótesis alterna $\mathrm{H} 1$ 'Existe influencia del lugar 
de la toma de muestra en el interior del bus sobre la contaminación microbiana" y se rechaza la hipótesis nula Ho.

Los sitios donde se lleva la toma de muestras tienen diferencias en cuanto al valor de la carga microbiana por lo tanto la contaminación en el interior del bus no es igual, el asidero vertical de aviso de parada presenta la mayor contaminación lo que se evidencia en la Figura 4.

Figura 4: Sitios de toma de muestras en el bus (base y asidero del asiento y asidero vertical).

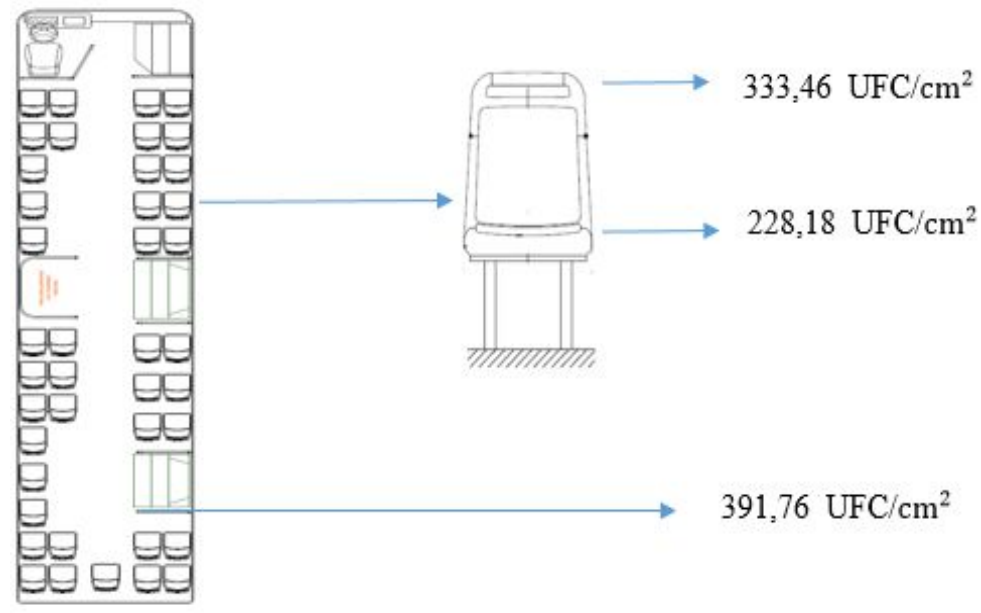

Fuente: Grupo de investigación.

Análisis inferencial entre el tipo de material del asidero vertical y la carga microbiana en la superficie: Se aplica la prueba ANOVA para determinar el estadístico de prueba para su comparación que se muestra en la tabla 6.

Tabla 6: Prueba ANOVA de un factor Lugar Material del asidero vertical vs. Carga microbiana.

\begin{tabular}{lll}
\hline F.V. & F & p valor \\
\hline Modelo & 0,68 & 0,5154 \\
Lugar de toma de la muestra & 0,68 & 0,5154 \\
\hline
\end{tabular}

Fuente: Grupo de Investigación.

Se aplica un nivel de significancia $=0,05$.

En el análisis de ANOVA se tiene un factor de nivel de significancia de 0,5154 que es mayor a 0,05 , por lo que se acepta la hipótesis alterna Ho "No existe influencia del tipo de material del asidero vertical sobre la contaminación microbiana en la superficie." La prueba de Tukey determina que no existe diferencia entre las medias de 
ninguno de los grupos, por lo tanto, la contaminación en todos los materiales estadísticamente es la misma.

Análisis inferencial entre la ruta y la carga microbiana en la superficie: Para el ritual de la significancia estadística para la hipótesis principal, se desarrolló un diseño experimental teniendo como criterio seleccionar dos rutas de las cinco para la comparación, que se evidencia en la tabla 7.

Tabla 7: Diseño experimental de la investigación.

\begin{tabular}{llll}
\hline Clasificación & Grupo 1 & Grupo 2 & p valor* \\
\hline Ruta & Ruta hospital & Ruta no hospital & $<0,0001$ \\
\hline
\end{tabular}

Fuente: Grupo de investigación.

Se presentan los estadísticos descriptivos para cada uno de los niveles de la variable independiente, así como para el total de la muestra. Se comprobó que existe diferencia significativa estadística entre el promedio de los grupos.

$*$ Se aplica un nivel de significancia $=0,05$.

En el análisis de T student para muestras independientes el nivel de significancia es 0,0001 es menor a 0.05, esto quiere decir que se acepta la hipótesis alterna H1 "En las rutas de transporte urbano que circulan por áreas de hospitales existe mayor contaminación ambiental en el asidero vertical de los buses de la ciudad de Ambato" y se rechaza la hipótesis nula Ho.

\section{Conclusiones.}

- La hipótesis general de la investigación demuestra que las rutas de transporte urbano que circulan por áreas de hospitales tienen niveles mayores de contaminación ambiental en el asidero vertical, base y asidero del asiento de los buses, en relación a las rutas que no circulan por hospitales; en condiciones adecuadas tanto del ambiente como de las personas, enfermedades como gripe, resfrío, influenza, pasando por alergias, dermatitis, conjuntivitis y tuberculosis se pueden contraer en el interior de los buses.

- Se identifica que en el 80\% (62/78) de cultivos hubo crecimiento de bacterias Cocos Gram Positivos, el 10\% (8/78) existió bacterias Bacilos Gram negativos mientras que el 10\% (8/78) no existió ningún tipo de crecimiento, si bien ambos tipos pueden causar enfermedades infecciosas estas se vuelven una preocupación para la salud pública cuando su estructura celular las hace resistentes a los antibióticos. 
- Luego de analizar los datos primarios del problema se establece la prevalencia que tiene la bacteria Staphylococcus aureus en relación a las otras encontradas, el resultado revelo que en aproximadamente el $60 \%$ de las unidades de transporte analizadas está presente esta bacteria, siendo potencialmente patógena en personas que se encuentran con sistemas inmunocomprometidos y que de no ser tratada a tiempo puede causar graves secuelas en la salud.

- Aplicado el análisis inferencial de la influencia del tipo de material del asidero vertical sobre los niveles de contaminación microbiológica, se determina que estadísticamente no existe incidencia de estas variables, es decir el aluminio, acero inoxidable y con recubrimiento polimérico tienen similares valores $(449,23 ; 456,25$ y 473,17 unidades formadoras de colonia por centímetro cuadrado respectivamente), se explica debido a que ninguno de los materiales descritos está comprobado que tenga propiedades antibacteriales (Alexander, 2009).

\section{Referencias bibliográficas.}

Alexander, J. W. (2009). History of the medical use of silver. Surgical Infections, 10(3), 289-292. doi:10.1089/sur.2008.9941

Conceicao, T., Diamantino, F., Coelho, C., de Lencastre, H., \& Aires-de-Sousa, M. (2013). Contamination of public buses with MRSA in Lisbon, Portugal: a possible transmission route of major MRSA clones within the community. PLoS One, 8(11), e77812. doi: 10.1371/journal.pone.0077812

Coia, J. E., Duckworth, G. J., Edwards, D. I., Farrington, M., Fry, C., Humphreys, H.,. Infection Control Nurses, A. (2006). Guidelines for the control and prevention of meticillin-resistant Staphylococcus aureus (MRSA) in healthcare facilities. J Hosp Infect, 63 Suppl 1, S1-44. doi: 10.1016/j.jhin.2006.01.001

Gaymard, A., Pichon, M., Degaud, M., Tasse, J., Dupieux, C., \& Laurent, F. (9 de Julio de 2016). Staphylococcus aureus resistente a la meticilina (MRSA) en medio ambiente de transporte público: datos del metropolitano de Lyon, Francia. American Journal of Infection Control. doi:10.1016/j.ajic.2014.08.016

Kassem, II. (2009). Concerning public transport as a reservoir of methicillin-resistant staphylococci. Lett Appl Microbiol, 48(2), 268. doi: 10.1111/j.1472765X.2008.02518.X

Lutz, J. K., van Balen, J., Crawford, J. M., Wilkins, J. R., Lee, J., Nava-Hoet, R. C., \& Hoet, A. E. (2014). Methicillin-resistant Staphylococcus aureus in public transportation vehicles (buses): Another piece to the epidemiologic puzzle. American Journal of Infection Control, 42(12), 1285-1290. doi: 10.1016/j.ajic.2014.08.016 
Otter, J. A., \& French, G. L. (2009). Bacterial contamination on touch surfaces in the public transport system and in public areas of a hospital in London. Lett Appl Microbiol, 49(6), 803-805. doi: 10.1111/j.1472-765X.2009. 02728.x

Peng, Y., Ou, Q., Lin, D., Xu, P., Li, Y., Ye, X., Yao, Z. (2015). Metro system in Guangzhou as a hazardous reservoir of methicillin-resistant Staphylococci: findings from a pointprevalence molecular epidemiologic study. Scientific Reports, 5(1). doi:10.1038/srep16087

Roberts, M. C., Soge, O. O., \& No, D. (2013). Comparison of Multi-Drug Resistant Environmental Methicillin-Resistant Staphylococcus aureus Isolated from Recreational Beaches and High Touch Surfaces in Built Environments. Front Microbiol, 4, 74. doi: 10.3389/fmicb.2013.00074 
Para citar el artículo indexado.

Galarza R., Espín S., Zaldumbide F., Tene O \& López I. (2018). Incidencia de las rutas del transporte público en la contaminación ambiental microbiana de buses urbanos Revista electrónica Ciencia Digital 2(2), 110-122. Recuperado desde: http://cienciadigital.org/revistacienciadigital2/index.php/CienciaDigital/article/view/76/71

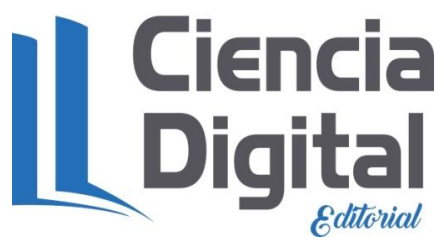

El artículo que se publica es de exclusiva responsabilidad de los autores y no necesariamente reflejan el pensamiento de la Revista Ciencia Digital.

El articulo queda en propiedad de la revista y, por tanto, su publicación parcial y/o total en otro medio tiene que ser autorizado por el director de la Revista Ciencia Digital.
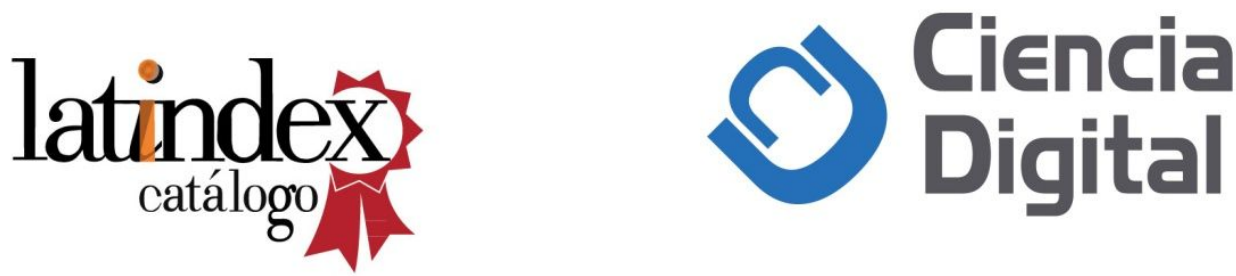